\title{
Vitamina D nativa nei pazienti con malattia renale cronica non in trattamento dialitico
}

\author{
Fabio Malberti \\ Divisione di Nefrologia e Dialisi, Istituti Ospitalieri, Cremona
}

\begin{abstract}
NATIVE VITAMIN D IN PATIENTS WITH CHRONIC KIDNEY DISEASE NOT TREATED WITH DIALYSIS Abstract. Vitamin D metabolism is involved in the regulation of bone metabolism and calcium homeostasis. Vitamin D receptors are, however, detectable in several tissues and organs not directly involved in the regulation of mineral metabolism; furthermore, not only bone cells, but also other cell types can synthesize active vitamin D. Altogether, these findings have suggested that vitamin D may have other potential effects in addition to the commonly known effects on mineral metabolism. Vitamin D deficiency can induce bone disease development and represents an important risk factor for neoplastic, autoimmune and cardiovascular diseases. This review focuses on the results of the main randomized trials on vitamin D supplementation in the general population and in patients with chronic kidney disease.
\end{abstract}

Key words: Vitamin D, Cholecalciferol, Calcifediol, Hyperparathyroidism, Bone fractures

Conflict of interest: None.

Ricevuto: 7 Maggio 2013; Accettato: 8 Maggio 2013

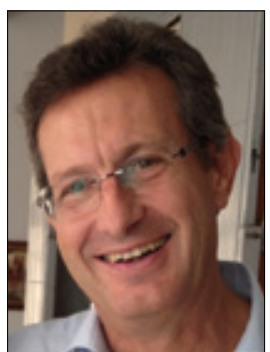

Il sistema ormonale della vitamina $\mathrm{D}$ è classicamente implicato nella regolazione dell'omeostasi calcica e del metabolismo osseo. La dimostrazione dell'esistenza di recettori per la vitamina D (VDR) in organi e tessuti non coinvolti direttamente nella regolazione del metabolismo minerale e l'osservazione che molte cellule sono in grado di sintetizzare la forma attiva di vitamina D (1.25-vitamina D) a partire dal precursore circolante (25-vitamina D) (1) hanno Fabio Malberti fatto supporre che la vitamina D possa avere altri effetti oltre $\mathrm{D}$ ha effetti antiproliferativi e che interviene nella modulazione della risposta immunitaria, nella regolazione del sistema renina-angiotensina (RAS) e nella regolazione della proliferazione delle cellule muscolari lisce della parete arteriosa e che può influenzare la funzione cardiaca e la funzione muscolare scheletrica (2-7). Inoltre, studi sperimentali hanno evidenziato che la vitamina $\mathrm{D}$ ha un effetto renoprotettivo sia nelle glomerulonefriti sperimentali sia nel danno renale indotto dall'iperglicemia (8-10).

Studi epidemiologici hanno documentato che il deficit di vitamina $\mathrm{D}$, oltre a indurre lo sviluppo di patologie ossee (rachitismo, osteomalacia, fratture ossee), è un importante fattore di rischio per lo sviluppo di numerose malattie croniche, quali neoplasie (colon, prostata, mammella), malattie autoimmuni (diabete mellito di tipo I, sclerosi multipla, malattia di Chron) e malattie cardiovascolari $(2,3,7)$. Recentemente, è stato documentato che il deficit di vitamina $\mathrm{D}$ è un importante predittore di progressione del danno renale e di mortalità nei pazienti con insufficienza renale cronica $(\mathrm{CKD})(11,12)$.

\section{Cenni di fisiologia}

Nei paesi occidentali, l'apporto alimentare di vitamina D è generalmente insufficiente per coprire il fabbisogno quotidiano $(20 \mu \mathrm{g} /$ die, $800 \mathrm{UI} / d i e)$ (13). Gli alimenti più ricchi di vitamina $\mathrm{D}\left(\mathrm{D}_{2}\right)$ sono alcuni pesci e derivati ittici (olio di fegato di merluzzo, salmone, pesce spada, sgombro), di consumo non frequente nei paesi occidentali, nei quali la principale fonte di vitamina $\mathrm{D}$ è data dall'esposizione ai raggi solari. I raggi ultravioletti $\mathrm{B}$ inducono la trasformazione a livello cutaneo del 7- e dell'8-diidrotachisterolo in pre-vitamina $\mathrm{D}_{3}$, che isomerizza con un processo temperatura-dipendente in vitamina $\mathrm{D}_{3}$ (o colecalciferolo). Una volta formatasi nei cheratinociti, la vitamina $\mathrm{D}_{3}$ viene liberata nei capillari del derma ed entra in circolo legata alla $D$ Binding Protein (DBP). La vitamina $\mathrm{D}_{3}$ è, poi, convertita nel fegato a 25 -vitamina $\mathrm{D}_{3}$ (calcifediolo) e quindi, ad opera dell'1-alfa idrossilasi (o CYP27B1) renale, nella forma attiva, la $1-25(\mathrm{OH})_{2}-\mathrm{D}_{3}$ (o calcitriolo). Il calcitriolo viene catabolizzato a metabolita inattivo dalla 24 -idrossilasi (o CYP24A1). La sintesi di calcitriolo è substrato-dipendente, per cui i livelli plasmatici saranno ridotti se c'è carenza di 25-vitamina $\mathrm{D}(25-\mathrm{D})$. La sintesi di calcitriolo è finemente regolata dall'attività della 1-alfa-idrossilasi e della 24-idrossilasi. Il paratormone (PTH), l'ipocalcemia e l'ipofosforemia 
stimolano l'attività dell'1-alfa-idrossilasi, mentre l'ipercalcemia, l'iperfosforemia e il FGF23 la inibiscono (1-3, 14). La 25-D ha un'emivita plasmatica di circa 15 giorni, mentre l'emivita del calcitriolo è di poche ore. I livelli plasmatici di 25-D sono indicativi del pool corporeo di vitamina D. Anche se non vi è unanimità sulla definizione dei livelli plasmatici normali di 25-D, in letteratura si parla di deficit di vitamina $\mathrm{D}$ quando $\mathrm{i}$ livelli di $25 \mathrm{D}$ sono inferiori a $30 \mathrm{ng} / \mathrm{mL}$ (75 nmol/L) (3). Sotto tali livelli si ha, infatti, un incremento del PTH in risposta alla riduzione dell'assorbimento intestinale di calcio (3). Pertanto, nella popolazione generale e nei pazienti con malattia renale cronica (CKD), è desiderabile mantenere i livelli plasmatici di 25-D al di sopra di $30 \mathrm{ng} / \mathrm{mL}(3,15,16)$. Si calcola che, nel 40$50 \%$ della popolazione generale, i livelli plasmatici siano $<30$ $\mathrm{ng} / \mathrm{mL}$ e che la prevalenza del deficit di vitamina $\mathrm{D}$ sia aumentata nell'ultima decade (3, 17-19)

\section{Deficit di vitamina D nella CKD}

Nei pazienti con CKD è abituale il riscontro di bassi livelli di calcitriolo, ma vi è anche un'elevata prevalenza di deficit di 25-D (20-22). Il deficit di 25-D è dovuto alla scarsa esposizione solare in pazienti spesso affetti da più comorbilità, alla ridotta sintesi di colecalciferolo a livello cutaneo, al ridotto apporto di alimenti contenenti vitamina $\mathrm{D}$, alle perdite urinarie di $25-\mathrm{D}$ e DBP in caso di proteinuria e alla riduzione della megalina, indispensabile per il riassorbimento tubulare della 25-D filtrata dal glomerulo $(3,23-25)$. Nella CKD, l'elevazione del FGF23 può contribuire alla riduzione dei livelli plasmatici di calcitriolo e di 25-D, per riduzione dell'attività dell'1-alfa-idrossilasi e per aumento dell'attività della 24-idrossilasi, che aumenta il catabolismo della 1-25 D e della 25-D in metaboliti inattivi $(1,26)$. Anche la 1.25-vitamina D stimola l'attività della 24-idrossilasi, per cui il trattamento con calcitriolo può contribuire a determinare i bassi livelli plasmatici di 25-D $(1,3)$.

\section{Deficit di vitamina D nella CKD e outcome}

Diversi studi hanno documentato che il deficit di vitamina D è un predittore indipendente di mortalità e di progressione dell'insufficienza renale nei pazienti con $\operatorname{CKD}(11,12,27,28)$. I vari studi concordano nel rilevare che, nei pazienti con CKD, si ha un aumento significativo del rischio relativo di progressione dell'insufficienza renale e di mortalità per livelli plasmatici di $25-\mathrm{D}$ inferiori a $15-17 \mathrm{ng} / \mathrm{mL}(11,12,27-31)$. Una recente metanalisi ha confermato che il deficit di vitamina $\mathrm{D}$ è un predittore indipendente di mortalità nei pazienti con CKD agli stadi 3 e 5 e nei pazienti in dialisi (32).

Recentemente, Urena et al. (33) hanno documentato in circa mille pazienti con CKD non in dialisi (eGFR medio $40 \mathrm{~mL} / \mathrm{min}$, età media 60 anni) che il deficit di vitamina $\mathrm{D}$ (livelli plasmatici di $25-\mathrm{D}<15 \mathrm{ng} / \mathrm{mL}$ ) si associa a una maggiore frequenza di iperparatiroidismo secondario $(\mathrm{PTH}>60 \mathrm{pg} / \mathrm{mL})$, di bassi livelli plasmatici di $1.25-\mathrm{D}(<16 \mathrm{pg} / \mathrm{mL})$ e di elevazione degli indici biochimici indicativi di un elevato turn-over osseo rispetto ai pazienti con normali livelli plasmatici di 25-D (>30 ng/mL). In sintesi, numerosi studi indicano in modo unanime che il deficit di vitamina D nei pazienti con CKD si associa sia a effetti negativi sul metabolismo minerale sia a un'evoluzione clinica negativa. I risultati di questi studi osservazionali fanno supporre che la correzione del deficit di vitamina D nei pazienti con CKD potrebbe avere effetti benefici non solo sul metabolismo minerale ma anche sulla progressione dell'insufficienza renale e sulla sopravvivenza.

\section{Effetti della supplementazione con vitamina D nella popolazione generale}

Non vi sono in letteratura studi randomizzati che abbiano valutato l'effetto del trattamento con vitamina D sulla mortalità. Autier et al. (34), in una metanalisi che includeva tutti gli studi randomizzati pubblicati fino al 2007 che riportavano i dati di mortalità nei pazienti trattati con vitamina $\mathrm{D}_{2} \mathrm{o} \mathrm{D}_{3}$ rispetto al placebo, hanno documentato un rischio relativo di mortalità significativamente inferiore nei pazienti trattati con vitamina D (RR 0.92, 95\%, IC: 0.86-0.99, p<0.001). Il limite della metanalisi è dato dal fatto che tutti gli studi considerati avevano come outcome primario o l'incidenza di fratture o la variazione della densità minerale ossea, mentre la sopravvivenza era un outcome secondario. LaCroix et al. (35), nel 2009, in uno studio randomizzato in doppio cieco in più di 36.000 donne in età postmenopausale, hanno documentato che la supplementazione con $400 \mathrm{U} /$ die di colecalciferolo associata a $1 \mathrm{~g}$ di calcio carbonato non riduce significativamente la mortalità $(\mathrm{RR} 0.91, \mathrm{IC}: 0.83-$ 1.01, p: NS) rispetto al placebo in un follow up medio di 7 anni. Dato che la vitamina $\mathrm{D}$ attiva (calcitriolo) è un inibitore diretto della sintesi di renina $(4,5)$, si è ipotizzato che il trattamento con vitamina $\mathrm{D}$ potesse ridurre i livelli di pressione arteriosa. Pittas et al. (36), in una metanalisi che ha considerato gli studi osservazionali e randomizzati che hanno valutato l'effetto della supplementazione della vitamina D sugli outcome cardiovascolari, hanno documentato una correlazione inversa tra i livelli plasmatici di 25-D e lo sviluppo di ipertensione arteriosa e di eventi cardiovascolari. Tuttavia, il trattamento con vitamina D (dosi giornaliere da 400 a $8.500 \mathrm{U}$ ) non modificava significativamente la pressione arteriosa. Nei 2 studi randomizzati che hanno valutato l'outcome cardiovascolare, la supplementazione con vitamina D non era superiore al placebo $(35,37)$.

Hypponen et al. (38) hanno dimostrato che la supplementazione con vitamina $\mathrm{D}_{3}$ nel primo anno di vita riduce il rischio di sviluppare diabete mellito di tipo I, in una popolazione (quella finlandese) in cui vi è un'elevata incidenza della malattia.

In una recente metanalisi, Bischopp-Ferrari et al. (39) hanno valutato l'effetto della supplementazione con vitamina D sulla prevenzione dello sviluppo di fratture ossee. Dati i risultati contrastanti presenti in letteratura, Bischopp-Ferrari et al. (39) hanno considerato solo gli studi randomizzati in doppio cieco che hanno valutato l'effetto della supplementazione con vitamina D (da sola o associata a supplementi di calcio) rispetto al placebo o alla sola supplementazione di calcio in soggetti di età superiore ai 65 anni. Rispettavano tali criteri 11 trial, che complessivamente avevano studiato 31.022 soggetti, che, nel follow up, sviluppavano 1.111 fratture femorali e 3.770 fratture non vertebrali. Il rischio di fratture era inversamente correlato ai livelli basali di $25-\mathrm{D}$ e il rischio relativo era significativamente inferiore per livelli plasmatici $>24 \mathrm{ng} / \mathrm{mL}$ 
rispetto a livelli $<24 \mathrm{ng} / \mathrm{mL}$ (39). Nell' analisi "intention to tre$a t$ ", il trattamento con vitamina $\mathrm{D}$ riduceva il rischio relativo di fratture non vertebrali in modo non significativo (fratture femorali: RR 0.90, IC: 0.80-1.01, p<0.07; fratture non vertebrali: RR 0.93, IC: 0.87-0.99, p<0.03). Considerando la dose reale di vitamina $\mathrm{D}_{3}$ assunta (piuttosto che la dose inizialmente assegnata), emergeva che la supplementazione riduceva significativamente il rischio di fratture (fratture femorali: RR 0.70, IC: $0.58-0.76, p<0.001$; fratture non vertebrali: HR 0.87, IC: $0.76-0.96, \mathrm{p}<0.007)$ solo se i dosaggi giornalieri erano uguali o superiori a $800 \mathrm{U}$ (39). I risultati della metanalisi sono in accordo con le recenti raccomandazioni dello IOM (Institute of Medicine) che raccomandano la supplementazione con $800 \mathrm{U} /$ die nei pazienti di età $>65$ anni con deficit di vitamina $\mathrm{D}$ e che suggeriscono di mantenere i livelli plasmatici di $25-\mathrm{D}$ a $>24$ $\mathrm{ng} / \mathrm{mL}(>60 \mathrm{nmol} / \mathrm{L})$ per ridurre il rischio di fratture $(13,39)$.

\section{Effetti della supplementazione con vitamina D nella CKD}

Non vi sono studi randomizzati che abbiano valutato l'effetto della supplementazione con vitamina D sulla sopravvivenza o sulla progressione dell'insufficienza renale nei pazienti con CKD.

Gli studi randomizzati finora pubblicati hanno valutato gli effetti della supplementazione su alcuni parametri del metabolismo minerale (in particolare, sul PTH) o sull'infiammazione. Kandula et al. (40), in una metanalisi che includeva 4 studi randomizzati che avevano valutato l'effetto della supplementazione con vitamina D sui livelli di PTH in pazienti con CKD e deficit di vitamina $\mathrm{D}$, hanno documentato che il trattamento con vitamina D naturale riduceva significativamente il PTH senza modificare la calcemia e la fosforemia. In uno studio recente condotto in una popolazione pediatrica (eGFR tra 15 e $70 \mathrm{~mL} /$ $\mathrm{min} / 1.73 \mathrm{~m}^{2} ; 25-\mathrm{D}<30 \mathrm{ng} / \mathrm{mL}$ ), la supplementazione con ergocalciferolo (dosi da 2.000 a $8.000 \mathrm{U} /$ die in relazione con i livelli basali di 25-D) preveniva lo sviluppo di iperparatiroidismo secondario rispetto al placebo nei 6 mesi di follow up, a parità di livelli di calcemia e fosforemia (41). Markmann et al. (42), in uno studio randomizzato della durata di 8 settimane, hanno dimostrato che il colecalciferolo $(40.000 \mathrm{U} /$ settimana, pari a 160 gocce) riduceva significativamente il $\mathrm{PTH}$ rispetto al placebo (a parità di livelli di calcemia) nei pazienti con CKD agli stadi 3 e 4 e deficit di vitamina D. La capacità della vitamina D nativa di ridurre il PTH è, ovviamente, inferiore rispetto a quella del metabolita attivo. Infatti, Kovesdy et al. (43), in uno studio randomizzato di 16 settimane in 80 pazienti con CKD agli stadi 3 e 4 e deficit di vitamina $\mathrm{D}$, hanno dimostrato che il paracalcitolo al dosaggio di 1-2 $\mu \mathrm{g} /$ die riduce il PTH significativamente rispetto all'ergocolecalciferolo (50.000 U settimanali/mensili). Alcuni studi, non randomizzati, hanno documentato che la correzione del deficit di vitamina D nei pazienti con CKD in dialisi migliora lo stato infiammatorio, riducendo i livelli di proteina $C$ reattiva e delle citochine flogogene $(44,45)$. In un recente studio randomizzato in doppio cieco condotto in pazienti diabetici con funzione renale normale, il trattamento con colecalciferolo $(1.000 \mathrm{U} /$ die $)$ per 8 settimane ha ridotto significativamente la PCR e ha aumentato i livelli di proteine antinfiammatorie (adiponectina) rispetto al placebo (46).

\section{Conclusioni}

La carenza di vitamina $\mathrm{D}$ è frequente nella popolazione generale e si associa a un aumentato rischio di osteoporosi, fratture, neoplasie, malattie autoimmuni ed eventi cardiovascolari. La correzione del deficit di vitamina $\mathrm{D}$ riduce significativamente il rischio di fratture non vertebrali nella popolazione generale (39). Uno studio osservazionale di 54 mesi su 422.822 soggetti di età $>45$ anni ha documentato che $\mathrm{i}$ livelli di $25-\mathrm{D}$ tra i 20 e i $36 \mathrm{ng} / \mathrm{mL}$ sono associati al minor rischio di mortalità e di morbilità per eventi cardiovascolari, rispetto a livelli plasmatici $<20$ $\mathrm{o}>36 \mathrm{ng} / \mathrm{mL}$ (47). I soggetti con livelli $<10 \mathrm{ng} / \mathrm{mL}$ avevano un RR (1.88, IC: 1.80-1.96) più elevato (47). Numerosi studi osservazionali hanno evidenziato che anche nei pazienti con CKD il deficit di vitamina $\mathrm{D}$ aumenta il rischio di mortalità e di progressione della CKD alla dialisi. Anche se, nei pazienti con CKD, mancano evidenze in merito al fatto che la supplementazione abbia un impatto sulla mortalità o sulla morbilità, la correzione del deficit è raccomandabile, in accordo con quanto suggerito dalle Linee Guida (15). Vari schemi terapeutici possono essere usati per normalizzare i livelli plasmatici di 25-D (2, 3, 13). In Italia sono disponibili il colecalciferolo e il calcifediolo, che possono essere somministrati con frequenza giornaliera (o settimanale) a un dosaggio di 3-4 gocce (750-1.000 U; $40 \mathrm{U}=1 \mathrm{ug}$ ) o di 20-30 gocce/settimana (5.000-7.500 U).

\section{Riassunto}

Il sistema ormonale della vitamina D è classicamente implicato nella regolazione dell'omeostasi calcica e del metabolismo osseo. L'esistenza di recettori per la vitamina D in organi e tessuti non coinvolti direttamente nella regolazione del metabolismo minerale e la capacità di molte cellule di sintetizzare la forma attiva di vitamina D dal precursore circolante hanno fatto supporre che la vitamina D possa avere altri effetti oltre ai classici effetti sul metabolismo minerale. Il deficit di vitamina D induce lo sviluppo di patologie ossee ed è un importante fattore di rischio per lo sviluppo di neoplasie, malattie autoimmuni e malattie cardiovascolari. In questa rassegna vengono esaminati i risultati dei principali studi randomizzati che hanno utilizzato la supplementazione con vitamina D nella popolazione generale e nei pazienti con insufficienza renale cronica.

Parole chiave: Vitamina D, Colecalciferolo, Calcifediolo, Iperparatiroidismo, Fratture ossee

Dichiarazione di conflitto di interessi: L'Autore dichiara di non avere conflitto di interesse.

Indirizzo degli Autori:

Fabio Malberti

Divisione di Nefrologia e Dialisi, Istituti Ospitalieri

Largo Priori 1

26100 Cremona

f.malberti@ospedale.cremona.it 


\section{Bibliografia}

1. Dusso AS, Brown AJ, Slatopolsky E. Vitamin D. Am J Physiol Ren Physiol 2005; 289: F8-28.

2. Holick MF. Resurrection of vitamin D deficiency and rickets. J Clin Invest 2006; 116: 2062-72.

3. Holick MF. Vitamin D deficiency. N Engl J Med 2007; 357: 266-81.

4. Li YC, Qiao G, Uskokovic M, et al. Vitamin D: A negative endocrine regulator of the renin-angiotensin system and blood pressure. J Steroid Biochem Mol Biol 2004; 89-90: 387-92.

5. Xiang W, Kong J, Chen S, et al. Cardiac hypertrophy in vitamin $\mathrm{D}$ receptor knockout mice: role of the systemic and cardiac renin-angiotensin systems. Am J Physiol Endocrinol Metab 2005; 288: E125-32.

6. Mitsuhashi T, Morris RC Jr, Ives HE. 1,25-dihydroxyvitamin D3 modulates growth of vascular smooth muscle cells. J Clin Invest 1991; 87: 1889-95.

7. Wang TJ, Pencina MJ, Booth SL, et al. Vitamin D deficiency and risk of cardiovascular disease. Circulation 2008; 117: 503-11.

8. Migliori M, Giovannini L, Panichi V, et al. Treatment with 1,25-Dihydroxyvitamin D3 preserves glomerular slit diaphragmassociated protein expression in experimental glomerulonephritis. Int J Immunopathol Pharmacol 2005; 18: 779-90.

9. Wang Y, Zhou J, Minto AW, et al. Altered vitamin D metabolism in type II diabetic mouse glomeruli may provide protection from diabetic nephropathy. Kidney Int 2006; 70: 882-91.

10. Zhang Z, Sun L, Wang Y, et al. Renoprotective role of the vitamin D receptor in diabetic nephropathy. Kidney Int 2008; 73 : 163-71.

11. Ravani P, Malberti F, Tripepi G, et al. Vitamin D levels and patients outcome in chronic kidney disease. Kidney Int 2009; 75: 88-95.

12. Melamed ML, Astor B, Michos ED, et al. 25-hydroxyvitamin $\mathrm{D}$ levels, race, and the progression of kidney disease. J Am Soc Nephrol 2009; 20: 2631-9.

13. Ross AC, Manson JE, Abrams SA, et al. The 2011 report on dietary reference intakes for calcium and vitamin $\mathrm{D}$ from the Institute of Medicine: what clinicians need to know. J Clin Endocrinol Metab 2011; 96: 53-8.

14. Wolf M. Update on fibroblast growth factor 23 in chronic kidney disease. Kidney Int 2012; 89: 737-47.

15. Kidney Disease: Improving Global Outcomes (KDIGO) CKDMBD Work Group. KDIGO clinical practice guideline for the diagnosis, evaluation, prevention, and treatment of chronic kidney disease-mineral and bone disorder (CKD-MBD). Kidney Int 2009; 76 (Suppl. 113): S1-130.

16. Bischoff-Ferrari HA, Giovannucci E, Willett WC, et al. Estimation of optimal serum concentrations of 25-hydroxyvitamin D for multiple health outcomes. Am J Clin Nutr 2006; 84: 18-28.

17. Zadshir A, Tareen N, Pan D, et al. The prevalence of hypovitaminosis D among US adults: data from NHANES III. Ethn Dis 2005; 15: S5-97-101.

18. Ginde AA, Liu MC, Camargo CA. Demographic differences and trends of vitamin D insufficiency in the US Population, 1988-2004. Arch Intern Med 2009; 169: 626-32.

19. Bodnar LM, Simhan HN, Powers RW, et al. High prevalence of vitamin D insufficiency in black and white pregnant women residing in the northern United States and their neonates. J Nutr
2007; 137: 447-52

20. Levin A, Bakris GL, Molitch M, et al. Prevalence of abnormal serum vitamin D, PTH, calcium, and phosphorus in patients with chronic kidney disease: results of the study to evaluate early kidney disease. Kidney Int 2007; 71: 31-8.

21. Isakova T, Wahl P, Vargas GS, et al. Fibroblast growth factor 23 is elevated before parathyroid hormone and phosphate in chronic kidney disease. Kidney Int 2011; 79: 1370-8.

22. Bhan I, Burnett-Bowie SA, Ye J, et al. Clinical measures identify vitamin D deficiency in dialysis. Clin J Am Soc Nephrol 2010; 5: 460-7.

23. Wesseling-Perry K, Salusky IB. Is replacement therapy with nutritional and active forms of vitamin $\mathrm{D}$ required in chronic kidney disease mineral and bone disorder? Curr Opin Nephrol Hypertens 2009; 18: 308-14.

24. Takemoto F, Shinki T, Yokoyama K, et al. Gene expression of vitamin $\mathrm{D}$ hydroxylase and megalin in the remnant kidney of nephrectomized rats. Kidney Int 2003; 64: 414-20.

25. De Boer IH, Ioannou GN, Kestenbaum B, et al. 25-Hydroxyvitamin D levels and albuminuria in the Third National Health and Nutrition Examination Survey (NHANES III). Am J Kidney Dis 2007; 50: 69-77.

26. Shimada T, Hasegawa H, Yamazaki Y, et al. FGF-23 is a potent regulator of vitamin D metabolism and phosphate homeostasis. J Bone Miner Res 2004; 19: 429-35.

27. Barreto DV, Barret FC, Liabeuf S, et al. Vitamin D affects survival independently of vascular calcification in chronic kidney disease. Clin J Am Soc Nephrol 2009; 4: 1128-1135.

28. Mehrotra R, Kermah DA, Salusky IB, et al. Chronic kidney disease,hypovitaminosis D, and mortality in the United States. Kidney Int 2009; 76: 977-983.

29. Jassal SK, Chonchol M, von Mühlen D, et al. Vitamin D, parathyroid hormone, and cardiovascularmortality in older adults: the Rancho Bernardo Study. Am J Med 2010; 123: 1114-20.

30. Navaneethan SD, Schold JD, Arrigain S, et al. Low 25-hydroxyvitamin D levels and mortality in non-dialysis-dependent CKD. Am J Kidney Dis 2011; 58 (4): 536-43.

31. Nakano C, Hamano T, Fujii N, et al. Combined use of vitamin D status and FGF23 for risk stratification of renal outcome. Clin J Am Soc Nephrol 2012; 7: 810-9.

32. Pilz S, Jodice S, Zittermann A, et al. Vitamin D status and mortality risk in CKD: a meta-analysis of prospective studies. Am J Kidney Dis 2011; 58: 374-82.

33. Ureña-Torres P, Metzger M, Haymann JP, et al. Association of kidney function, vitamin $\mathrm{D}$ deficiency, and circulating markers of mineral and bone disorders in CKD. Am J Kidney Dis 2011; 58: 544-553.

34. Autier P, Gandini S. Vitamin D supplementation and total mortality: a meta-analysis of randomized controlled trials. Arch Intern Med 2007; 167: 1730-7.

35. LaCroix AZ, Kotchen J, Anderson G, et al. Calcium plus vitamin D supplementation and mortality in postmenopausal women: the Women's Health Initiative calcium-vitamin D randomized controlled trial. J Gerontol A Biol Sci Med Sci 2009; 64 (5): 559-67.

36. Pittas AG, Chung M, Trikalinos T, et al. Systematic review: Vitamin D and cardiometabolic outcomes. Ann Inter Med 2010; 152: 307-14.

37. Hsia J, Heiss G, Ren H, et al. Calcium/vitamin D supplementa- 
tion and cardiovascularevents. Circulation 2007; 115: 846-54.

38. Hyppönen E, Läärä E, Reunanen A, Järvelin MR, Virtanen SM. Intake of vitamin $\mathrm{D}$ and risk of type 1 diabetes: a birth-cohort study. Lancet 2001; 358: 1500-3.

39. Bischoff-Ferrari HA, Willett WC, Orav EJ, et al. A pooled analysis of vitamin $\mathrm{D}$ dose requirements for fracture prevention. N Engl J Med 2012; 367: 40-9.

40. Kandula P, Dobre M, Schold JD, et al. Vitamin D supplementation in chronic kidney disease: a systematic review and metaanalysis of observational studies and randomized controlled trials. Clin J Am Soc Nephrol 2011; 6: 50-62.

41. Shroff R, Wan M, Gullett A, et al. Ergocalciferol supplementation in children with CKD delays the onset of secondary hyperparathyroidism: a randomized trial. Clin J Am Soc Nephrol 2012; 7: 216-23.

42. Markmann P, Agerskov H, Thineshkumar S, et al. Randomized controlled trial of cholecalciferol supplementation in chronic kidney disease patients with hypovitaminosis D. Nephrol Dial Transplant 2012; 27: 3523-31.

43. Kovesdy CP, Lu JL, Malakauskas SM, et al. Paricalcitol ver- sus ergocalciferol for secondary hyperparathyroidism in CKD stages 3 and 4: a randomized controlled trial. Am J Kidney Dis 2012; 59: 58-66.

44. Stubbs JR, Idiculla A, Slusser J, et al. Cholecalciferol supplementation alters calcitriol-responsive monocyte proteins and decreases inflammatory cytokines in ESRD. J Am Soc Nephrol 2010; 21: 353-61.

45. Matias PJ, Jorge C, Ferreira C, et al. Cholecalciferol supplementation in hemodialysis patients: effects on mineral metabolism, inflammation, and cardiac dimension parameters. Clin J Am Soc Nephrol 2010; 5: 905-11.

46. Neyestani TR, Nikooyeh B, Alavi-Majd H. Improvement of vitamin D status via daily intake of fortified yogurt drink either with or without extra calcium ameliorates systemic inflammatory biomarkers, including adipokines, in the subjects with type 2 diabetes. J Clin Endocrinol Metab 2012; 97: 2005-11.

47. Drior Y, Giveon S, Hoshen M, et al. Vitamin D levels for preventing acute coronary syndrome and mortality: evidence of a non-linear association. J Clin Endocrinol Metab 2013; doi: 10.1210/jc2013-1185. 